\title{
Neoadjuvant Treatment With Trastuzumab and FOLFOX Induces a Complete Pathologic Response in a Metastatic ERBB2 (HER2)-Amplified Duodenal Cancer
}

\author{
Ahmad Hamad, MD; ; Aatur D. Singhi, MD, PhD ; Nathan Bahary, MDc; Kevin McGrath, MD; \\ Rula Amarin, MD; ${ }^{\mathrm{e}}$; Herbert J. Zeh, MDa; and Amer H. Zureikat, MD
}

\begin{abstract}
Overexpression of HER2 protein and amplification of the ERBB2 gene has been observed in various adenocarcinomas, providing a therapeutic target that can be used to extend the survival of a select cohort of patients. Anti-HER2 therapy has been successfully applied to gastric and colorectal cancers, but its use and potential benefit in small intestinal carcinomas is not well characterized. We applied antiHER2 therapy to an ERBB2-amplified advanced duodenal adenocarcinoma, adding trastuzumab to FOLFOX in the neoadjuvant setting. A 61-year-old woman with an advanced duodenal cancer harboring an ERBB2 amplification received preoperative trastuzumab and FOLFOX. Restaging revealed significant tumor downstaging with no metastasis. After multidisciplinary assessment, she underwent pancreaticoduodenectomy. Final pathologic analysis revealed no residual invasive adenocarcinoma, consistent with a complete neoadjuvant treatment response. This case report emphasizes the need for further molecular characterization of small bowel cancers; genetic alterations may provide therapeutic targets to improve the prognosis of these rare and aggressive malignancies.
\end{abstract}

J Nat/ Compr Canc Netw 2017;15(8):983-988 doi:10.6004/jnccn.2017.0140

Small bowel adenocarcinoma (SBA) is a rare tumor that accounts for $37 \%$ of small bowel malignancies and $<2 \%$ of all gastrointestinal tumors. ${ }^{1}$ Although surgery remains the mainstay of therapy, most patients present with advanced disease, and 5-year overall survival (OS) rates range from $26 \%$ to $40 \% .^{2}$ Because of its low incidence and lack of available trials, current chemotherapy regimens used for adjuvant or metastatic SBA are extrapolated from colorectal cancer (CRC) and gastric cancer, with poor results. ${ }^{3}$ Overexpression of the HER2 protein and amplification of the ERBB2 gene has been observed in various adenocarcinomas, providing a therapeutic target that can be used to extend the survival of

From the aDivision of Surgical Oncology, bepartment of Surgical Pathology, 'Department of Medical Oncology, and dDivision of Gastroenterology, University of Pittsburgh Medical Center, Pittsburgh, Pennsylvania; and eDepartment of Medical Oncology, King Hussein Cancer Center, Amman, Jordan.

Submitted March 13, 2017; accepted for publication May 12, 2017. a select cohort of patients. Anti-HER2 therapy has been successfully applied to gastric cancer and CRC, but its use and potential benefit in small intestinal carcinomas is not well characterized. 4,5 We applied anti-HER2 therapy to an ERBB2-amplified advanced duodenal adenocarcinoma, adding trastuzumab to FOLFOX in the neoadjuvant setting, to achieve a complete pathologic response $(\mathrm{cPR})$ in the resected specimen.

\section{Case Report}

A 61-year-old woman of Middle Eastern descent known to have hypertension, hyperlipidemia, and gastroesophageal reflux disease presented with a 4 -month history

The authors have disclosed that they have no financial interests, arrangements, affiliations, or commercial interests with the manufacturers of any products discussed in this article or their competitors.

Correspondence: Amer H. Zureikat, MD, UPMC Pancreatic Cancer Center, Division of Surgical Oncology, University of Pittsburgh Medical Center, 5150 Centre Avenue, Suite 421, Pittsburgh, PA 15232.

E-mail: zureikatah@upmc.edu 
Hamad et al

of epigastric discomfort. Due to persistent symptoms, she underwent a right upper-quadrant ultrasound that demonstrated a distended gallbladder without stones, and a markedly dilated common bile duct with no stones or filling defects (Figure 1). These findings prompted an MRI, which revealed a dilated intrahepatic and extrahepatic biliary tree, dilation of the common bile duct to the preampullary region, no pancreatic mass, and possible duodenal wall thickening. Subsequent upper endoscopy demonstrated a malignant-appearing, fungating, ulcerated, and partially obstructive circumferential mass in the second and third portions of the duodenum (Figure 2).

Biopsies of the abnormal duodenal mucosa revealed an invasive, moderately differentiated adenocarcinoma. Molecular analysis of the duodenal biopsy demonstrated the tumor to be HER2-positive (immunohistochemistry [IHC] +2 with $50 \%$ staining of tumor cells and fluorescence in situ hybridization [FISH]-amplified), microsatellite stable, and negative for ALK and PD-L1 (Figure 3). Further molecular testing using next-generation sequencing identified no mutations in BRAF, HRAS, KRAS, NRAS, and PIK3CA. A colonoscopy showed a tubular adenoma, which was resected. A staging CT scan of the chest, abdomen, and pelvis showed irregular thickening involving parts of the second and third parts of the duodenum and multiple enlarged regional lymph nodes, but no evidence of metastatic disease. Laboratory studies revealed a normal CBC count, metabolic profile, liver function tests, CA19. 9, and carcinoembryonic antigen (CEA) levels.

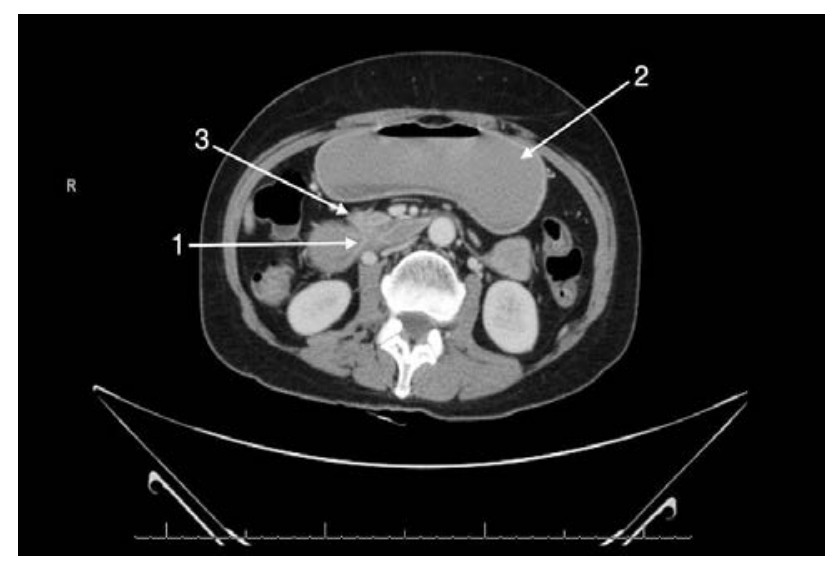

Figure 1. CT image of 61-year-old patient with duodenal adenocarcinoma showing (1) duodenal cancer, (2) distended stomach, and (3) the pancreas.

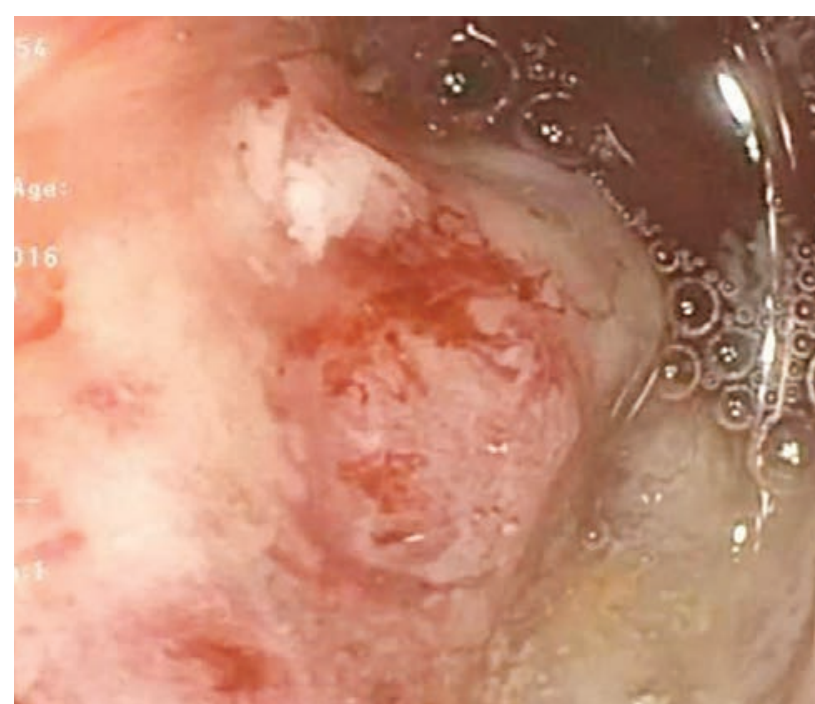

Figure 2. Endoscopic image showing a near circumferential duodenal adenocarcinoma.

The patient was recommended to undergo a robotic-assisted classic pancreaticoduodenectomy (Whipple) procedure at the University of Pittsburgh Medical Center. At laparoscopy, exploration revealed the presence of a segment $4 \mathrm{~B}$ subcentimeter liver lesion. Frozen section analysis confirmed it to be metastatic duodenal adenocarcinoma and the resection was aborted. No other lesions were noted on laparoscopy. Molecular analysis of the liver biopsy was consistent with the primary tumor, demonstrating it to be microsatellite stable, PD-L1 expressionnegative, ALK-negative by IHC, and HER2-positive (IHC +2 with $50 \%$ staining of tumor cells, and FISH-positive).

After multidisciplinary discussion, and due to the presence of an ERBB2 amplification, the patient was recommended to undergo treatment with 6 cycles of FOLFOX and trastuzumab, followed by a potential pancreaticoduodenectomy in the absence of new metastatic disease on restaging. Because the management of SBA is mostly extrapolated from $\mathrm{CRC}$, the rationale was to treat this oligometastatic SBA with "neoadjuvant"/definitive chemotherapy followed by resection in the absence of new metastasis; a strategy analogous to the treatment of metastatic CRC. Before initiation of therapy, a PET scan confirmed the FDG-avid duodenal primary with regional lymphadenopathy and no metastasis. The patient commenced therapy with FOLFOX (oxaliplatin, $85 \mathrm{mg} / \mathrm{m}^{2}$; leucovorin, $400 \mathrm{mg} / \mathrm{m}^{2}$; 5FU bolus, 400 


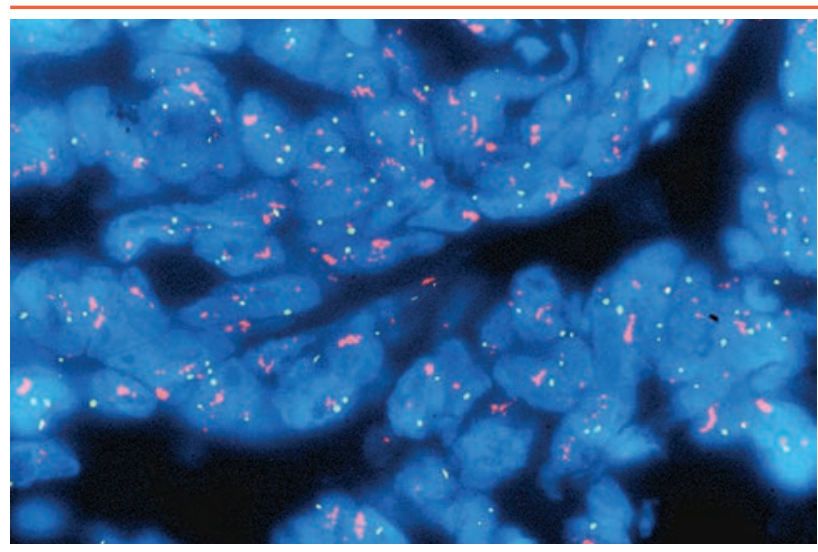

Figure 3. HER2 amplification status by fluorescence in situ hybridization. Green dots represent centromere 17 and red dots represent HER2. Note evidence of HER2 amplification indicated by large number of red dots.

$\mathrm{mg} / \mathrm{m}^{2}$; and infusional $5 \mathrm{FU}, 2,400 \mathrm{mg} / \mathrm{m}^{2}$ on days 1 and 14) and trastuzumab (6 mg/kg loading dose on day 1 of the first cycle followed by $4 \mathrm{mg} / \mathrm{kg}$ on day 1 and 14 days from the second cycle onwards). Notably, the trastuzumab dose used in this patient was lower than that used in the ToGA trial $(8 \mathrm{mg} / \mathrm{kg}$ loading, followed by $6 \mathrm{mg} / \mathrm{kg}$ maintenance every 3 weeks) due to the 2-weekly FOLFOX treatment schedule as opposed to the 3-weekly capecitabine/cisplatin schedule, respectively. Therapy was interrupted due to the need for a duodenal stent placement for obstructive symptoms and removal of her subclavian chemotherapy port due to catheter tip thrombosis. After 4 cycles of FOLFOX and trastuzumab, restaging PET/CT revealed a marked decrease in the thickening and FDG avidity of the duodenal primary, resolution of FDG avidity of the regional lymph nodes, and no evidence of metastatic disease. After multidisciplinary discussion, a decision was made to reexplore and resect the primary tumor.

At exploration, no metastases were identified, and the patient underwent a robotic-assisted classic pancreaticoduodenectomy with resection of the segment 4B liver scar (old biopsy site). Final pathologic analysis revealed no residual invasive adenocarcinoma, consistent with a complete neoadjuvant treatment response (Figure 4). All surgical margins were free of neoplasia, and 39 resected lymph nodes were benign. The liver segment was also free of neoplasia, and final stage was ypTONO. Her postoperative course was uneventful, and she was discharged on the seventh postoperative day.

\section{Discussion}

SBA is a rare aggressive malignancy with a poor prognosis. Although surgical resection represents the best chance for cure, most patients present with advanced disease; in a large study, $40.6 \%$ and $24 \%$ presented with $\mathrm{T} 4$ tumors or distant metastasis, respectively. ${ }^{1}$ In the absence of randomized clinical trials, treatment options for advanced and metastatic SBA have been extrapolated from CRC, with poor results. Although several studies have reported improved response rates using $5 \mathrm{FU}$ in combination with oxaliplatin, irinotecan, or cisplatin, complete responses are rare and OS remains poor (Table 1). Moreover, although molecular profiling has allowed the application of targeted therapeutics in CRC, a significant lag in the molecular understanding of SBA remains, highlighting the need to identify target pathways that could improve patient prognosis. ${ }^{6,7}$ In this novel case report, we successfully used preoperative trastuzumab in combination with FOLFOX in an ERBB2-amplified metastatic duodenal cancer to achieve a cPR in the surgical specimen. Although the contribution of anti-HER2 therapy to this patient's favorable PR is difficult to quantify, it is noteworthy that complete tumor regression was observed after only 4 cycles of trastuzumab and FOLFOX.

Anti-HER2 therapy is being increasingly used in various gastrointestinal malignancies. As a member of the epidermal growth factor receptor family, HER2 is located on chromosome $17 \mathrm{q} 21$ and acts as a proto-oncogene. It is a cell membrane receptor tyrosine kinase that is activated following ligand binding and receptor dimerization. ${ }^{8}$ Dimerization

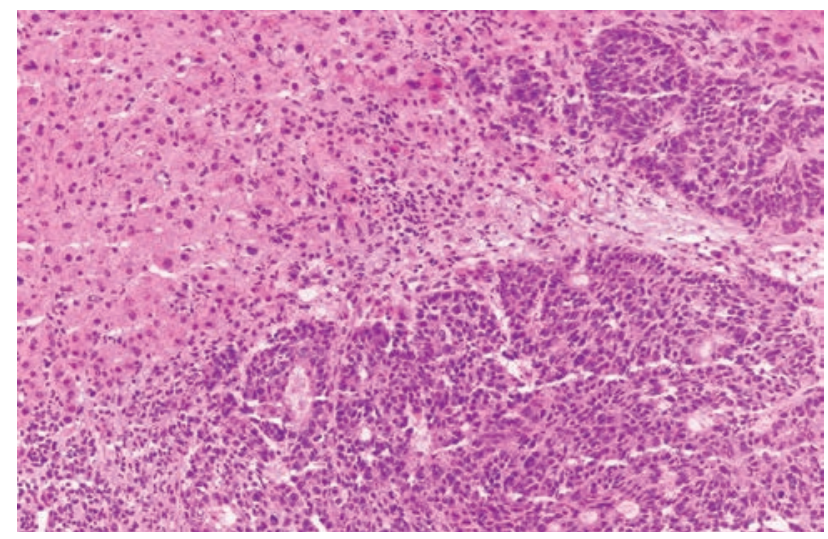

Figure 4. Microscopic pathology image of patient's metastatic duodenal adenocarcinoma in the liver (hematoxylin and eosin, original magnification $\times 200$ ). 
Hamad et al

\begin{tabular}{|c|c|c|c|c|c|}
\hline Author/Year & Study Type & $\begin{array}{l}\text { Patients } \\
\text { (n) }\end{array}$ & Chemotherapy Regimen & $\begin{array}{c}\text { Response } \\
\text { Rate } \\
(\%)\end{array}$ & $\begin{array}{l}\text { Median OS } \\
\quad(\mathrm{mo})\end{array}$ \\
\hline Rochlin et al, ${ }^{17} 1965$ & Retrospective & 11 & 5FU monotherapy & 36 & NR \\
\hline Ouriel \& Adams, ${ }^{18} 1984$ & Retrospective & 14 & 5FU-based & NR & 10.7 \\
\hline Jigyasu et al,, 1984 & Retrospective & 14 & 5FU-based & 7 & 9 \\
\hline Gibson et al, ${ }^{20} 2005$ & Prospective phase II & 38 & $5 \mathrm{FU}+$ doxorubicin + mitomycin & 18 & 8 \\
\hline Locher et al, ${ }^{21} 2005$ & Retrospective & 20 & $5 F U$ + platinum & 21 & 14 \\
\hline Fishman et al, 222006 & Retrospective & 44 & Various agents & 29 & 18.6 \\
\hline Czaykowski \& Hui, ${ }^{3} 2007$ & Retrospective & 16 & 5FU-based & 6 & 15.6 \\
\hline \multirow[t]{2}{*}{ Overman et al, ${ }^{23} 2008$} & \multirow[t]{2}{*}{ Retrospective } & 29 & $5 F U+$ platinum & 41 & 14.8 \\
\hline & & 41 & Various agents & 17 & 12 \\
\hline Ono et al, ${ }^{24} 2008$ & Retrospective & 10 & Cisplatin + irinotecan & 12.5 & 17.3 \\
\hline Overman et al, ${ }^{25} 2009$ & Prospective phase II & 30 & CAPOX & 50 & 20.4 \\
\hline Suenaga et al, ${ }^{26} 2009$ & Retrospective & 10 & 5FU single agent & 10 & 12 \\
\hline \multirow[t]{4}{*}{ Zaanan et al, ${ }^{27} 2010$} & \multirow[t]{4}{*}{ Retrospective } & 48 & FOLFOX & 34 & 17.8 \\
\hline & & 10 & LV/5FU & 0 & 13.5 \\
\hline & & 19 & LV/5FU + cisplatin & 30 & 9.6 \\
\hline & & 16 & FOLFIRI & 9 & 10.6 \\
\hline Onkendi et al, ${ }^{15} 2012$ & Retrospective & 10 & Various agents & NR & 19 \\
\hline Xiang et $\mathrm{al}^{28} 2012$ & Prospective phase II & 33 & Modified FOLFOX & 48.5 & 15.2 \\
\hline McWilliams et al, ${ }^{29} 2012$ & Prospective phase II & 23 & CAPOX + irinotecan & 39 & 12.7 \\
\hline \multirow[t]{5}{*}{ Tsushima et al, ${ }^{30} 2012$} & \multirow[t]{5}{*}{ Retrospective } & 60 & $5 F U+$ monotherapy & 20 & 13.9 \\
\hline & & 17 & 5FU + cisplatin & 38 & 12.6 \\
\hline & & 22 & 5FU + oxaliplatin & 42 & 22.2 \\
\hline & & 11 & $5 \mathrm{FU}+$ irinotecan & 25 & 9.4 \\
\hline & & 22 & Other agents & 21 & 8.1 \\
\hline Gulhati et al, ${ }^{31} 2017$ & Prospective phase II & $30^{\mathrm{b}}$ & CAPOX + bevacizumab & 48.3 & 12.9 \\
\hline Total & - & 596 & - & 25.9 & 13.6 \\
\hline
\end{tabular}

Abbreviations: 5FU, 5-fluorouracil; CAPOX, capecitabine/oxaliplatin; FOLFOX, 5FU/oxaliplatin/leucovorin; FOLFIRI, 5FU/irinotecan/leucovorin; LV, leucovorin; NR, not reported; OS, overall survival.

aStudies with sample size $<10$ were excluded.

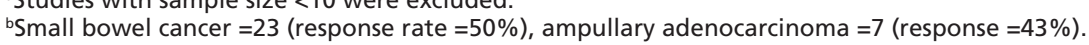

causes transphosphorylation of the receptor kinase domains, which recruits molecules responsible for downstream signaling pathways. ${ }^{6}$ These receptors function through different pathways to regulate cell motility, proliferation, differentiation, migration, and apoptosis. ${ }^{8}$ Amplification of ERBB2 has been implicated in the development of gastric, ovarian, breast, prostate, lung, and colorectal cancers. ${ }^{6,8} \mathrm{~A}$ recent trial examining the combination of trastuzumab with chemotherapy (cisplatin and 5FU or capecitabine) in patients with HER2-positive metastatic gastric cancer (ToGA trial) showed improved median OS compared with chemotherapy alone (13.8 vs 11.1 months, respectively; $P=.0046){ }^{4,9}$ More recently, the HERACLES trial showed that $30 \%$ of pretreated patients with HER2-mutated metastatic CRC achieved an objective response, whereas 59\% achieved disease stabilization after treatment with trastuzumab and the tyrosine kinase inhibitor lapatinib. ${ }^{5,10}$

However, although HER2 protein overexpression has been reported in $8.2 \%$ to $53.4 \%$ of gastric adenocarcinomas (ToGA trial: gastric, $21 \%$; gastroesophageal, $2 \%$ ) and $5 \%$ of CRCs, its frequency in small intestinal adenocarcinomas is only approximately $2.1 \% .^{2,6,11}$ In a study by Overman et al, ${ }^{12}$ only 1 of 54 cases of small intestinal adenocarcinoma ex- 
hibited HER2 protein expression. Likewise, Chan et $\mathrm{al}^{13}$ reported that 47 of 49 tumors completely lacked HER2 protein expression by IHC. Although the remaining 2 cases showed a $1+$ HER2 staining pattern, none exhibited HER2 gene amplification by FISH. In 51 cases of small intestinal adenocarcinomas, Aparicio et $\mathrm{al}^{14}$ found that only 2 cases showed HER2 expression, and both were located in the ileum, whereas 32 patients with duodenal adenocarcinoma lacked any HER2 expression.

Despite the low incidence of ERBB2 amplification in SBA, the increased availability and feasibility of molecular testing coupled with potential therapeutic benefits of targeted therapy may be worthwhile in this genetically defined subgroup, particularly in the absence of large trials to guide therapy. In our patient, testing for ERBB2 amplification expression was performed because none of the other known drivers of SBA where found in her primary lesion. Therefore, our report highlights the need to molecularly profile select SBAs and to test for ERBB2 amplification, particularly when known mutations are not implicated in the pathogenesis.

cPR to neoadjuvant therapy in resected duodenal cancer is uncommon; however, neoadjuvant therapy in this disease entity is not widely used compared with other gastrointestinal cancers. Onkendi et $\mathrm{al}^{15}$ reviewed the Mayo Clinic experience with salvage neoadjuvant therapy for unresectable/recurrent (nonmetastatic) duodenal adenocarcinoma and observed a cPR in 2 of 10 cases, whereas Kelsey et $\mathrm{al}^{16}$ observed a cPR in 2 of 11 patients treated with neoadjuvant chemoradiation for localized duodenal cancer. In our patient, because the added benefit of anti-ERBB2 therapy in achieving this patient's cPR remains unknown, she was recommended to undergo postoperative therapy with FOLFOX and trastuzumab, followed by trastuzumab monotherapy at a dose of $6 \mathrm{mg} / \mathrm{kg}$ every 3 weeks for a total of 1 year (extrapolated from the adjuvant treatment of breast cancer). Notably, at 6 months post resection, her CT scan showed no evidence of recurrent disease.

\section{Conclusions}

This case report emphasizes the need for further molecular characterization of small bowel cancers; genetic alterations may provide therapeutic targets to improve the prognosis of these rare and aggressive malignancies.

\section{References}

1. Bilimoria KY, Bentrem DJ, Wayne JD, et al. Small bowel cancer in the United States: changes in epidemiology, treatment, and survival over the last 20 years. Ann Surg 2009;249:63-71.

2. Gu MJ, Hong SM, Jung SJ; Korean Small Intestinal Cancer Study Group. HER2 protein expression and HER2 gene amplification are infrequent in small intestinal carcinomas. Virchows Arch 2013;462:603-607.

3. Czaykowski P, Hui D. Chemotherapy in small bowel adenocarcinoma: 10year experience of the British Columbia Cancer Agency. Clin Oncol (R Coll Radiol) 2007;19:143-149.

4. Bang YJ, Van Cutsem E, Feyereislova A, et al. Trastuzumab in combination with chemotherapy versus chemotherapy alone for treatment of HER2 positive advanced gastric or gastro-oesophageal junction cancer (ToGA) a phase 3, open-label, randomised controlled trial. Lancet 2010;376:687697.

5. Sartore-Bianchi A, Trusolino L, Martino C, et al. Dual-targeted therapy with trastuzumab and lapatinib in treatment-refractory, KRAS codon 12/13 wild-type, HER2-positive metastatic colorectal cancer (HERACLES): a proof-of-concept, multicentre, open-label, phase 2 trial. Lancet Oncol 2016;17:738-746.

6. Braga VM, de Oliveira MB, Netto CC, et al. Human epidermal growth factor receptor 2-positive duodenal adenocarcinoma: a case report and review of the literature. Case Rep Oncol 2015;8:285-289.

7. Dabaja BS, Suki D, Pro B, et al. Adenocarcinoma of the small bowel: presentation, prognostic factors, and outcome of 217 patients. Cancer 2004;101:518-526.

8. Montemurro F, Scaltriti M. Biomarkers of drugs targeting HER-family signalling in cancer. J Pathol 2014;232:219-229.

9. Albarello L, Pecciarini L, Doglioni C. HER2 testing in gastric cancer. Adv Anat Pathol 2011;18:53-59.

10. Sorscher SM. Marked response to single agent trastuzumab in a patient with metastatic HER-2 gene amplified rectal cancer. Cancer Invest 2011;29:456-459.

11. Valtorta E, Martino C, Sartore-Bianchi A, et al. Assessment of a HER2 scoring system for colorectal cancer: results from a validation study. Mod Pathol 2015;28:1481-1491.

12. Overman MJ, Pozadzides J, Kopetz S, et al. Immunophenotype and molecular characterisation of adenocarcinoma of the small intestine. $\mathrm{Br} \mathrm{J}$ Cancer 2010;102:144-150.

13. Chan OT, Chen ZM, Chung F, et al. Lack of HER2 overexpression and amplification in small intestinal adenocarcinoma. Am J Clin Pathol 2010;134:880-885.

14. Aparicio T, Svrcek M, Zaanan A, et al. Small bowel adenocarcinoma phenotyping, a clinicobiological prognostic study. Br J Cancer 2013;109:3057-3066.

15. Onkendi EO, Boostrom SY, Sarr MG, et al. Neoadjuvant treatment of duodenal adenocarcinoma: a rescue strategy. J Gastrointest Surg 2012;16:320-324.

16. Kelsey CR, Nelson JW, Willett CG, et al. Duodenal adenocarcinoma: patterns of failure after resection and the role of chemoradiotherapy. Int J Radiat Oncol Biol Phys 2007;69:1436-1441

17. Rochlin DB, Smart CR, Silva A. Chemotherapy of malignancies of the gastrointestinal tract. Am J Surg 1965;109:43-46.

18. Ouriel K, Adams JT. Adenocarcinoma of the small intestine. Am J Surg 1984;147:66-71

19. Jigyasu D, Bedikian AY, Stroehlein JR. Chemotherapy for primary adenocarcinoma of the small bowel. Cancer 1984;53:23-25.

20. Gibson MK, Holcroft CA, Kvols LK, Haller D. Phase II study of 5-fluorouracil, doxorubicin, and mitomycin $\mathrm{C}$ for metastatic small bowel adenocarcinoma. Oncologist 2005;10:132-137.

21. Locher C, Malka D, Boige V, et al. Combination chemotherapy in advanced small bowel adenocarcinoma. Oncology 2005;69:290-294. 
Hamad et al

22. Fishman PN, Pond GR, Moore MJ, et al. Natural history and chemotherapy effectiveness for advanced adenocarcinoma of the small bowel: a retrospective review of 113 cases. Am J Clin Oncol 2006;29:225-231.

23. Overman MJ, Kopetz S, Wen S, et al. Chemotherapy with 5-fluorouracil and a platinum compound improves outcomes in metastatic small bowel adenocarcinoma. Cancer 2008;113:2038-2045.

24. Ono M, Shirao K, Takashima A, et al. Combination chemotherapy with cisplatin and irinotecan in patients with adenocarcinoma of the small intestine. Gastric Cancer 2008;11:201-205.

25. Overman MJ, Varadhachary GR, Kopetz S, et al. Phase II study of capecitabine and oxaliplatin for advanced adenocarcinoma of the small bowel and ampulla of Vater. J Clin Oncol 2009;27:2598-2603.

26. Suenaga M, Mizunuma N, Chin K, et al. Chemotherapy for small-bowel adenocarcinoma at a single institution. Surg Today 2009;39:27-31.

27. Zaanan A, Costes L, Gauthier M, et al. Chemotherapy of advanced small-bowel adenocarcinoma: a multicenter AGEO study. Ann Oncol 2010;21:1786-1793.
28. Xiang XJ, Liu YW, Zhang L, et al. A phase II study of modified FOLFOX as first-line chemotherapy in advanced small bowel adenocarcinoma. Anticancer Drugs 2012;23:561-566.

29. McWilliams RR, Mahoney MR, Marchello BT, et al. Pharmacogenetic dosing by UGT1A1 genotype as first-line therapy for advanced small-bowel adenocarcinoma: a North Central Cancer Treatment Group (NCCTG) trial [abstract]. J Clin Oncol 2012;30(Suppl):Abstract 314

30. Tsushima T, Taguri M, Honma Y, et al. Multicenter retrospective study of 132 patients with unresectable small bowel adenocarcinoma treated with chemotherapy. Oncologist 2012;17:1163-1170.

31. Gulhati P, Raghav K, Shroff RT, et al. Bevacizumab combined with capecitabine and oxaliplatin in patients with advanced adenocarcinoma of the small bowel or ampulla of vater: a single-center, open-label, phase 2 study. Cancer 2017;123:1011-1017.

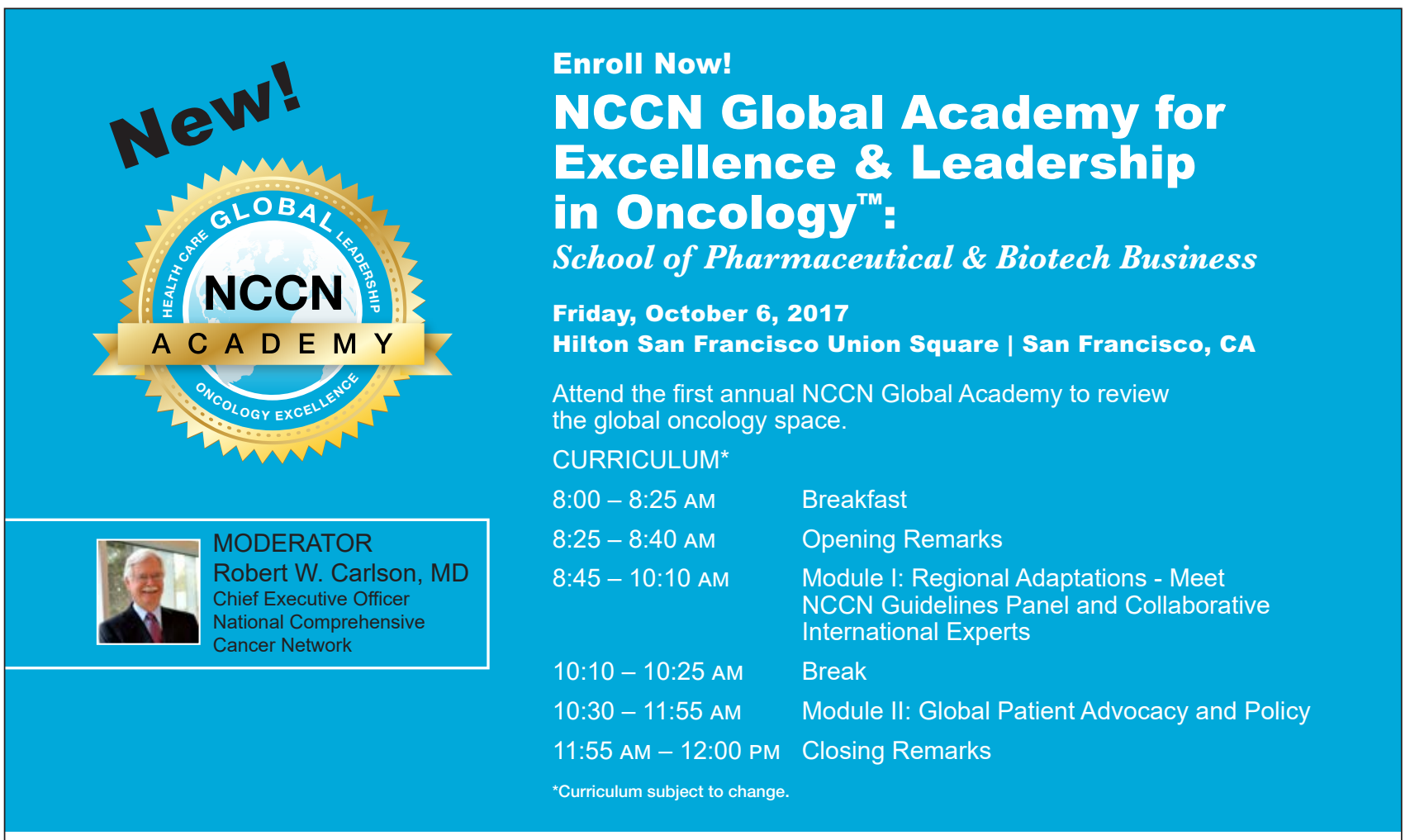

Visit NCCN.org/academy to enroll and access the complete program curriculum.

JNCCN-N-0242-0817 